\author{
Military Technical College \\ Kobry El-Kobbah, \\ Cairo, Egypt.
}

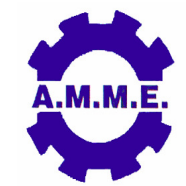

\title{
EFFECTS OF MIXING CHAMBER SHAPE ON CUTTING PERFORMANCE IN ABRASIVE WATER JET
}

\author{
H-R Lee*, Y-K Kwak ${ }^{\star *}, \mathrm{H}-\mathrm{Y} \mathrm{Kim}^{\star * \star}$ and J-H Ahn ${ }^{\star \star \star *}$
}

\begin{abstract}
It is well known that abrasive water jet (AWJ) was developed as a kind of highdensity energy processing technologies. AWJ is used to obtain cutting quality of various materials such as metal, ceramics, glass and composite materials within a short manufacturing time because of the characteristics of heatless and noncontact processing. However, surface roughness and dimension error like round, burr, taper depend on the cutting conditions such as pump pressure, cutting speed, orifice diameter, standoff distance, abrasive flow rate and work piece.

In this paper, the effect of the shape of mixing chamber on surface quality is studied. Parabolic mixing chamber is proposed and performance is compared to that of cylindrical mixing chamber by experiment. The surface roughness was improving $0.15 \mu \mathrm{m}$ to $2.29 \mu \mathrm{m}$ and the taper angle was improving $0.0716^{\circ}$ to $0.143^{\circ}$ by parabolic mixing chamber.
\end{abstract}

\section{KEY WORDS}

AWJ (Abrasive Water-Jet), Cutting Head, Mixing Chamber, Kerf taper, Surface Roughness.

\footnotetext{
* Graduate student, Intelligent Control and Automation Division, Dept. Mechanical Eng., Pusan National University, Busan, South Korea.

** Graduate student, Dept. of Mechanical and Intelligence System Engineering, Pusan National University, Busan, South Korea.

*** Associate professor, Research Institute of Mechanical Technology, Pusan National University, Busan, South Korea.

**** Professor, Dept. Mechanical Engineering, Pusan National University and ERC/NSDM, Busan, South Korea.
} 


\title{
NOMENCLATURE
}

\author{
$W_{T} \quad$ Cutting width at top of workpiece \\ $W_{B} \quad$ Cutting width at bottom of workpiece \\ $T \quad$ Thickness of workpiece
}

\section{INTRODUCTION}

Greater advances have been noticed in the material, which are harder and difficult to machine. The traditional methods have become ineffective for machining of hard and brittle materials such that productivity cannot be achieved. Due to this some nontraditional machining processes came into existence and the latest addition to that series is the abrasive water jet (AWJ).

In abrasive water jet, a small stream of fine grained abrasive particles is mixed in suitable proportion, which is focused on a workpiece surface through a nozzle. Material removal occurs due to erosion caused by the impact of abrasive particles on the work surface. Non-contact of the tool with work piece, no heat affected zone, low machining forces on the work surface and ability to machine a wide range of materials has increased the use of abrasive water jet machining over other machining processes [1].

However, the cutting quality of this technology, such as the kerf taper, characterized by a wider entry at the top than the exit at the bottom, is one of the major obstructions that limit its applications. In the last decades, a considerable research effort has been made towards understanding and minimizing the kerf taper in AWJ cut components. This includes the study of the jet dynamic characteristics, and the kerf geometrical features with respect to the process and jetting parameters. It has been found that in order to minimize the kerf taper angle, low jet traverse speeds are normally selected at high water pressures. Such a selection of the traverse speed is not preferred in practice as it reduces the cutting rate and increases the process costs. Studies have also been reported to use other cutting techniques, such as controlled nozzle oscillation and multipass cutting operations to reduce kerf taper and improve other cutting performance measures [2].

In this paper, the effect of the shape of mixing chamber on surface quality is carried out. New shape of mixing chamber is proposed and performance is compared to that of commercialized cylindrical mixing chamber by experiment.

\section{DEGINE OF MIXING CHAMBER}

Figure 1 shows a cross sectional view of the AWJ cutting head. An AWJ cutting head consist the following parts: focusing tube, orifice, mixing chamber, water channel, air and abrasive channel, cutting head body. The water channel and the abrasive channel is combined into the mixing chamber where high speed water jet mixes with air and abrasive. The focusing tube guides the water jet to the cutting point and helps to transfer the kinetic energy to abrasive particles as well as to mix with water. 
Md. G. Mostofa [3] showed that the vortex created inside the mixing chamber by CFD simulation. Inside the mixing chamber air possess very high turbulence as the air comes in contact with high speed water jet. As air carries the abrasive so the streamlines of air are very important because experimental investigations of $M$. Hashish suggested that this turbulence is responsible for entrainment of the abrasive particle inside the high speed water jet. From the result of the air stream line, the vortex phenomenon provides the initial mechanism of abrasive entrainment. Fig. 2. shows the vortex created inside the mixing chamber by water, air, abrasive. To control the stream line and to increase the abrasive entrainment parabolic shapes of mixing chamber was designed and applied for cutting. If the prediction is correct then there will be difference in the cutting performance. The parabolic and cylindrical mixing chambers are shown in the Fig. 3.

\section{EXPERIMENTS}

\section{Experimental Setup}

Figure 4 shows the abrasive water jet machine (TOPS, SJA-1530) which was used for this experiment. The experimental conditions for two types of mixing chambers are shown in Table 1. Two types of mixing chamber were used simultaneously and have cut a workpiece. The cutting performance evaluated by a taper angel and surface roughness on workpiece. Figure 5 shows the surface roughness measurement gives the reflection of the cutting. For surface roughness measurement each workpiece was two points at the lower $3 \mathrm{~mm}$ of top, upper $3 \mathrm{~mm}$ of bottom and Stylus (SJ-301, Mitutoyo). Surface roughness was measured three times and average value was used.

The kerf geometry of a through cut generated by abrasive water jets may be described as in Fig. 6 . It is characterized by a small rounded corner at the top edge owing to the plastic deformation of material caused by jet energy. The kerf is wider at the top than at the bottom. In addition, the plastically deformed material rolls over at the bottom of the kerf forming burrs at the jet exit side when cutting ductile materials [4]. The kerf taper angle for each cut was calculated using the measured values of the top and bottom width for each cut based on the equation [5].

$$
\text { Taper angle }=\tan ^{-1}\left(\frac{W_{T}-W_{B}}{2 T}\right)
$$

\section{Results}

The measurement data for two types of mixing chamber are shown in Table 2, and Table 3. This indicates that for a same applied pressure, abrasive flow rate, cutting speed and material.

In Figure 7, taper angle data of each condition for different mixing chamber was plotted against the variation of abrasive flow rate. The plotted trend shows that the taper angle values are decreasing with the increase in abrasive flow rate. Figure 8 describes the surface roughness variation corresponding to abrasive flow rate for changing the design of mixing chamber. Surface roughness varies with the change 
of abrasive flow rate. Abrasive flow rate increases then the surface roughness becomes decrease for same cutting speed and same mixing chamber. For any flow rate of abrasive, surface roughness obtained by using parabolic mixing chamber is minimum. So from the appearance it can be concluded that the parabolic mixing chamber is better for mixing in abrasive water jet cutting head. From the overall evaluation it can be observed that, parabolic mixing chamber is more efficient in mixing air, water and abrasive. Two types of investigation were done to observe the mixing quality for different shapes of mixing chamber.

\section{CONCLUSIONS}

In this research, parabolic mixing chamber is proposed and cutting performance is compared to that of cylindrical mixing chamber by experiment. The major results are summarized as follow:

1. The surface roughness was improve $0.15 \mu \mathrm{m}$ to $2.29 \mu \mathrm{m}$ and the taper angle was improve $0.0716^{\circ}$ to $0.143^{\circ}$ by using parabolic mixing chamber.

2. Parabolic shaped mixing chamber was proved more efficient in mixing air, water and abrasive.

\section{REFERENCES}

[1] Mahabalesh Palleda, "A study of taper angles and material removal rates of drilled holes in the abrasive water jet machining process", Journal of Materials Processing Technology, Vol.189, pp 292-295, (2007).

[2] D.K. Shanmugam, J. Wang and H. Liu, "Minimization of kerf tapers in abrasive water jet machining of alumina ceramics using a compensation technique", International Journal of Machine Tools \& Manufacture, Vol. 48, pp1527-1534, (2008).

[3] Md. G. Mostofa, Y. K. Kwak and J. H. Ahn, "Computational fluid analysis of abrasive waterjet cutting head", Journal of Mechanical Science and Technology, Vol. 24, pp 249-252, (2010)

[4] J. Wang, "Abrasive Water jet Machining of Polymer Matrix Composites - Cutting Performance, Erosive process and Predictive Models", The International Journal of Advanced manufacturing Technology, Vol. 15, pp 757-768,(1999)

[5] Ahmet Hascalik, Ulas Caydas, Hakan Curun, "Effect of traverse speed on abrasive water jet machining of Ti-6Al-4V alloy", Material and Design, Vol. 28, pp 1953-1957, (2007) 


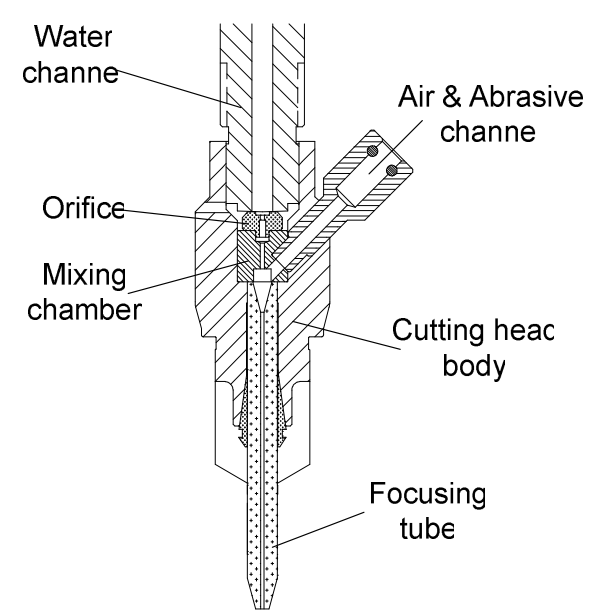

Fig. 1. Structure of the AWJ cutting head

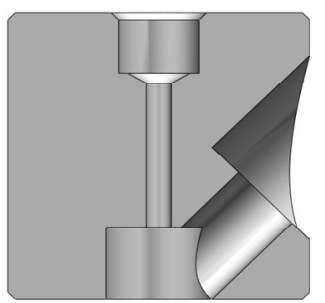

(a) Cylindrical type

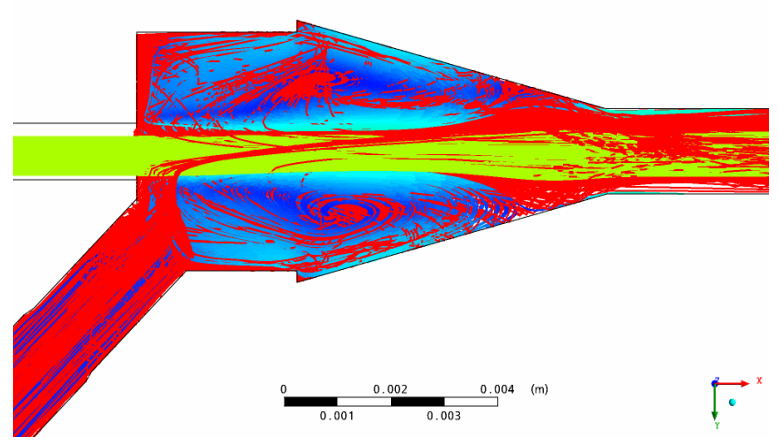

Fig. 2. Mixing of water, air, abrasive.

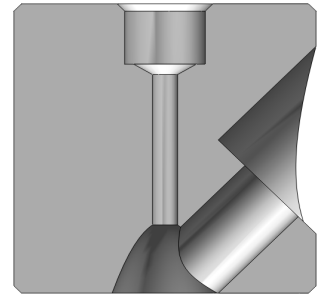

(b) Parabolic type

Fig.3. Two types of mixing chamber geometry.

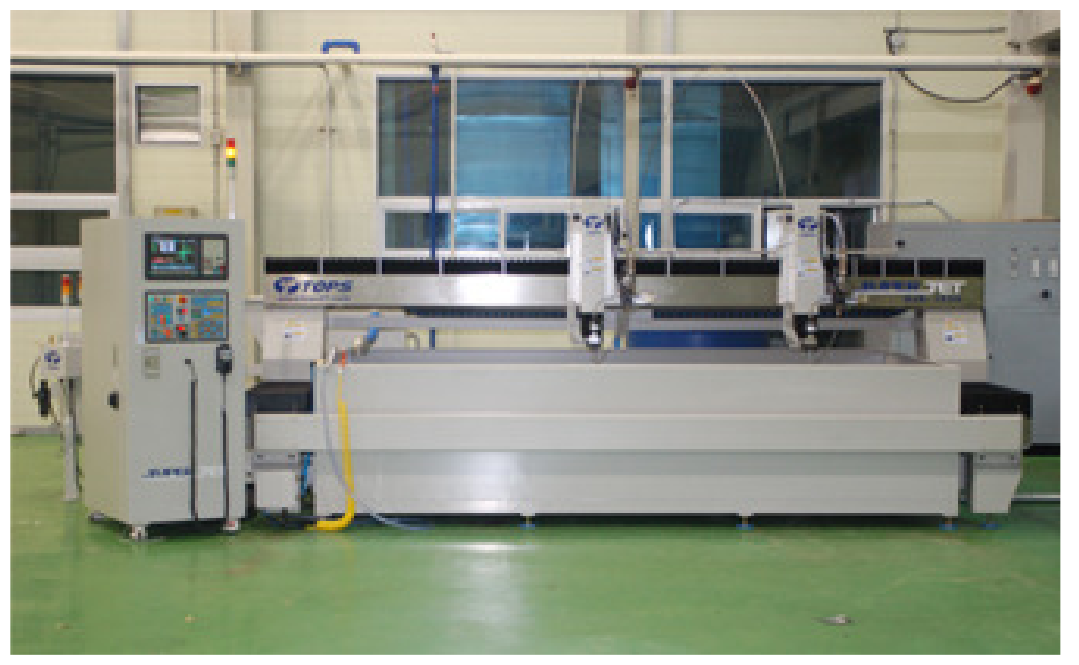

Fig. 4. Abrasive water jet cutting system (SJA-1530). 


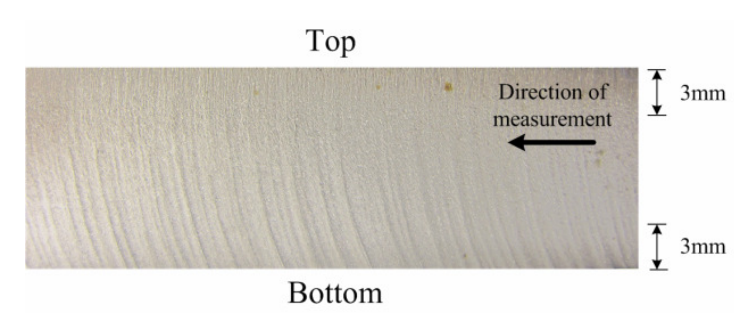

Fig. 5. Photograph of cutting surface and measurement point.

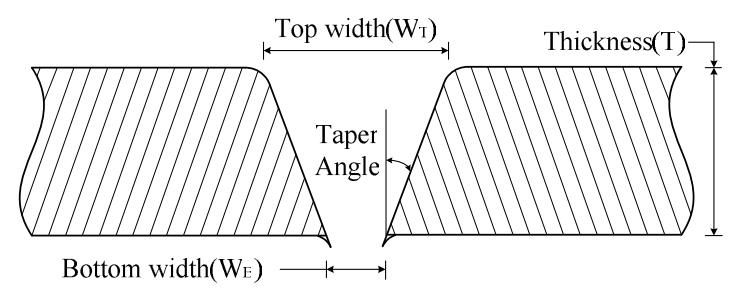

Fig. 6. Schematic and definition of kerf geometry and measurement taper angle.

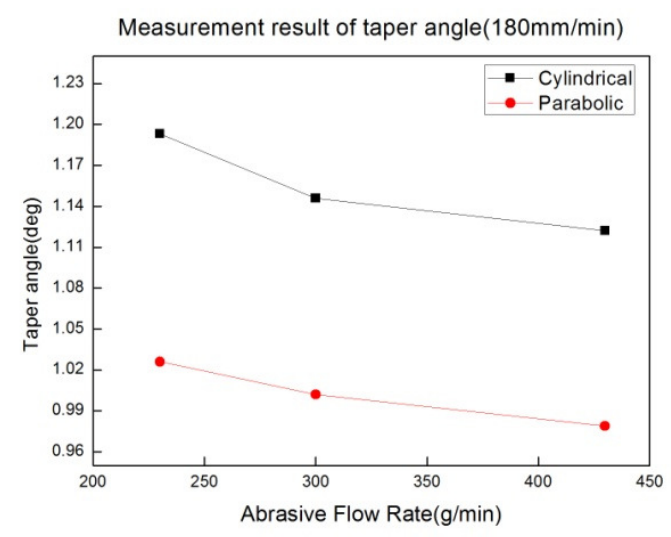

Fig. 7. Taper angle in workpiece corresponding to the change in abrasive flow rate.
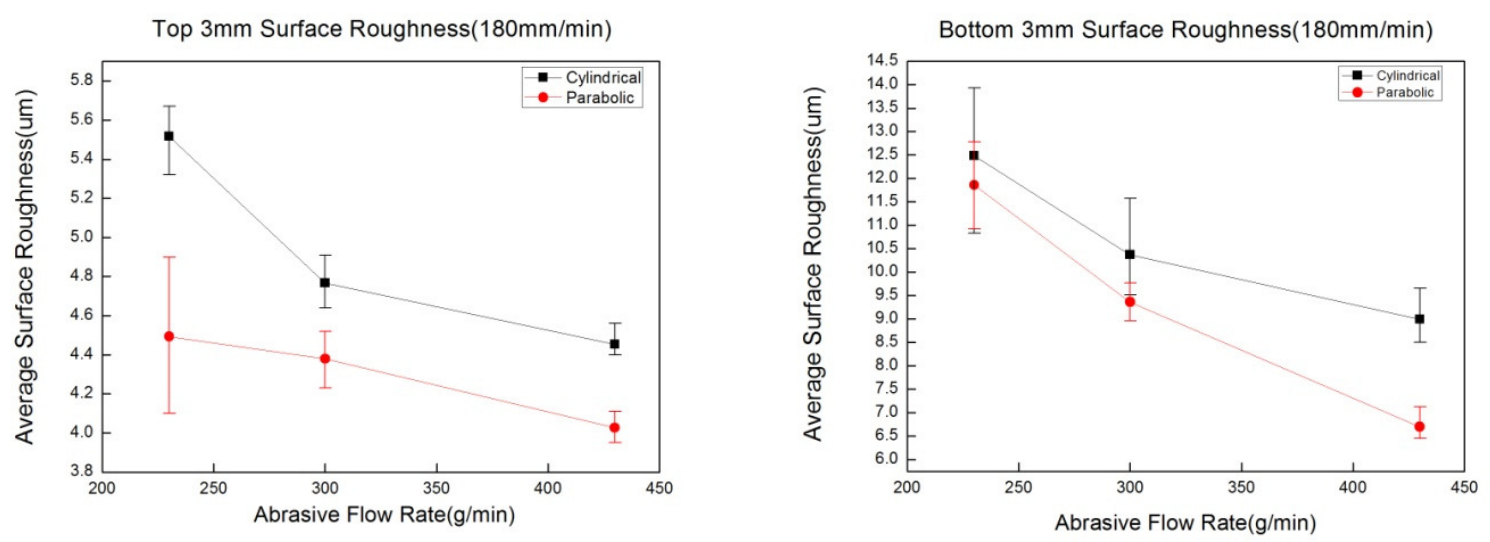

Fig.8. Variations of surface roughness in different point corresponding to the change in abrasive flow rate 
Table 1. Experimental conditions.

\begin{tabular}{|c|c|}
\hline Parameters & Conditions \\
\hline Cutting pressure & $3,800 \mathrm{bar}$ \\
\hline Abrasive flow rate & $230,300,430 \mathrm{~g} / \mathrm{min}$ \\
\hline Abrasive material & Garnet $(\# 80)$ \\
\hline Cutting speed & $90,180 \mathrm{~mm} / \mathrm{min}$ \\
\hline Stand off distance & $1.2 \mathrm{~mm}$ \\
\hline Orifice diameter & $0.33 \mathrm{~mm}$ \\
\hline Workpiece material & SUS \\
\hline Workpiece thickness & $12 \mathrm{~mm}$ \\
\hline Nozzle diameter & $1.01 \mathrm{~mm}$ \\
\hline Nozzle length & $76.2 \mathrm{~mm}$ \\
\hline Mixing chamber type & Cylindrical, Parabolic \\
\hline
\end{tabular}

Table 2. Taper and average surface roughness for cylindrical mixing chamber.

\begin{tabular}{|c|c|c|c|c|c|}
\hline \multirow{2}{*}{ No. } & Cutting Speed & Abrasive flow rate & Taper & \multicolumn{2}{|c|}{ Surface roughness $(\mu \mathrm{m})$} \\
\cline { 5 - 6 } & $(\mathrm{mm} / \mathrm{min})$ & $(\mathrm{g} / \mathrm{min})$ & angle $\left({ }^{\circ}\right)$ & Top $3 \mathrm{~mm}$ & Bottom 3mm \\
\hline 1 & 90 & 230 & 0.740 & 3.987 & 6.64 \\
\hline 2 & 90 & 300 & 0.716 & 3.837 & 5.49 \\
\hline 3 & 90 & 430 & 0.692 & 3.803 & 5.060 \\
\hline 4 & 180 & 230 & 1.193 & 5.517 & 12.487 \\
\hline 5 & 180 & 300 & 1.146 & 4.767 & 10.367 \\
\hline 6 & 180 & 430 & 1.122 & 4.453 & 8.993 \\
\hline
\end{tabular}

Table 3. Taper and average surface roughness for parabolic mixing chamber.

\begin{tabular}{|c|c|c|c|c|c|}
\hline \multirow{2}{*}{ No. } & Cutting Speed & Abrasive flow rate & Taper & \multicolumn{2}{|c|}{ Surface roughness $(\mu \mathrm{m})$} \\
\cline { 5 - 6 } & $(\mathrm{mm} / \mathrm{min})$ & $(\mathrm{g} / \mathrm{min})$ & angle $\left({ }^{\circ}\right)$ & Top $3 \mathrm{~mm}$ & Bottom $3 \mathrm{~mm}$ \\
\hline 1 & 90 & 230 & 0.644 & 3.830 & 6.290 \\
\hline 2 & 90 & 300 & 0.621 & 3.547 & 5.567 \\
\hline 3 & 90 & 430 & 0.621 & 3.340 & 4.910 \\
\hline 4 & 180 & 230 & 1.026 & 4.493 & 11.86 \\
\hline 5 & 180 & 300 & 1.002 & 4.380 & 9.367 \\
\hline 6 & 180 & 430 & 0.979 & 4.027 & 6.700 \\
\hline
\end{tabular}

